\title{
Preoperative Deltoid Size and Fatty Infiltration of the Deltoid and Rotator Cuff Correlate to Outcomes After Reverse Total Shoulder Arthroplasty
}

\author{
Brett P. Wiater MD, Denise M. Koueiter MS, Tristan Maerz MS, \\ James E. Moravek Jr MD, Samuel Yonan DO, David R. Marcantonio MD, \\ J. Michael Wiater MD
}

Received: 28 March 2014 / Accepted: 29 October 2014/Published online: 12 November 2014

(C) The Association of Bone and Joint Surgeons (B) 2014

\begin{abstract}
Background Reverse total shoulder arthroplasty (RTSA) allows the deltoid to substitute for the nonfunctioning rotator cuff. To date, it is unknown whether preoperative deltoid and rotator cuff parameters correlate with clinical outcomes.

Questions/purposes We asked whether associations exist between 2-year postoperative results (ROM, strength, and outcomes scores) and preoperative (1) deltoid size; (2) fatty infiltration of the deltoid; and/or (3) fatty infiltration of the rotator cuff.
\end{abstract}

\footnotetext{
One of the authors certifies that he (JMW), or a member of his immediate family, has or may receive payments or benefits, during the study period, an amount of USD 10,000-100,000 from Zimmer, Inc (Warsaw, IN, USA) and USD 10,000-100,000 from Synthes, Inc (West Chester, PA). The institution of the authors has received, during the study period, funding in an amount of USD 10,000100,000 from OMEGA, USD 10,000-100,000 from OREF, USD 10,000-100,000 from Zimmer, Inc (Warsaw, IN, USA), and USD 10,000-100,000 from Tornier (Edina, MN, USA).

All ICMJE Conflict of Interest Forms for authors and Clinical Orthopaedics and Related Research ${ }^{\circledR}$ editors and board members are on file with the publication and can be viewed on request.

Clinical Orthopaedics and Related Research ${ }^{\mathbb{R}}$ neither advocates nor endorses the use of any treatment, drug, or device. Readers are encouraged to always seek additional information, including FDA-approval status, of any drug or device prior to clinical use. Each author certifies that his or her institution approved the human protocol for this investigation, that all investigations were conducted in conformity with ethical principles of research.
}

B. P. Wiater, J. E. Moravek Jr J. M. Wiater ( $\square)$ Department of Orthopaedic Surgery, Beaumont Health System, 3601 W 13 Mile Road, Royal Oak, MI 48073, USA

e-mail: mwiater@beaumont.edu; jmwiater@aol.com

D. M. Koueiter, T. Maerz

Department of Orthopaedic Research, Beaumont Health System, Royal Oak, MI, USA
Methods A prospective RTSA registry was reviewed for patients with cuff tear arthropathy or massive rotator cuff tears, minimum 2-year followup, and preoperative shoulder MRI. Final analysis included 30 patients (average age, $71 \pm 10$ years; eight males, 22 females). Only a small proportion of patients who received an RTSA at our center met inclusion and minimum followup requirements (30 of 222; 14\%); however, these patients were found to be similar at baseline to the overall group of patients who underwent surgery in terms of age, gender, and preoperative outcomes scores. The cross-sectional area of the anterior, middle, and posterior deltoid was measured on axial proton density-weighted MRI. Fatty infiltration of the deltoid, supraspinatus, infraspinatus, teres minor, and subscapularis were quantitatively assessed on sagittal T1weighted MR images. Patients were followed for ConstantMurley score, American Shoulder and Elbow Surgeons (ASES) scores, subjective shoulder value, pain, ROM, and strength. Correlations of muscle parameters with all outcomes measures were calculated.

Results Preoperative deltoid size correlated positively with postoperative Constant-Murley score $(67.27 \pm 13.07)$ $(\rho=0.432, \quad p=0.017)$, ASES $(82.64 \pm 14.25) \quad(\rho=$ $0.377 ; \mathrm{p}=0.40)$, subjective shoulder value $(82.67 \pm$ 17.89) $\quad(\rho=0.427 ; \quad \mathrm{p}=0.019), \quad$ and strength $(3.72$ pounds \pm 2.99 pounds $)(\rho=0.454 ; p=0.015)$. Quantitative deltoid fatty infiltration $(7.91 \% \pm 4.32 \%)$ correlated

S. Yonan, D. R. Marcantonio

Department of Radiology, Beaumont Health System, Royal Oak, MI, USA 
with decreased postoperative ASES scores $(\rho=-0.401$; $\mathrm{p}=0.047$ ). Quantitative fatty infiltration of the infraspinatus $(30.47 \% \pm 15.01 \%)$ correlated with decreased postoperative external rotation $\left(34.13^{\circ} \pm 16.80^{\circ}\right)$ ( $\rho=-0.494 ; p=0.037)$.

Conclusions Larger preoperative deltoid size correlates with improved validated outcomes scores, whereas fatty infiltration of the deltoid and infraspinatus may have deleterious effects on validated outcomes scores and ROM after RTSA. The current study is a preliminary exploration of this topic; future studies should include prospective enrollment and standardized MRI with a multivariate statistical approach. Quantitative information attained from preoperative imaging not only holds diagnostic value, but, should future studies confirm our findings, also might provide prognostic value. This information may prove beneficial in preoperative patient counseling and might aid preoperative and postoperative decision-making by identifying subpopulations of patients who may benefit by therapy aimed at improving muscle properties.

Level of Evidence Level III, prognostic study.

\section{Introduction}

Reverse total shoulder arthroplasty (RTSA) has become instrumental in relieving pain and returning function to patients with end-stage rotator cuff disease. It allows the deltoid to substitute for a nonfunctioning rotator cuff, and multiple studies have shown that it results in changes in muscle recruitment, tension, and force distribution in the deltoid and remaining rotator cuff muscles compared with the native shoulder $[1-3,12,13,19,24]$. RTSA increases the efficiency of the deltoid as a forward elevator and abductor by increasing its tension and lever arm with a distalized and medialized center of rotation coupled with a semiconstrained articulation. In addition, the anterior and posterior deltoid fibers are recruited more for abduction and forward elevation at the expense of rotation owing to the muscle fiber force vectors assuming a more vertical position [1, 2, 5]. The loss of the contribution of the anterior deltoid to internal rotation may be compensated for by the remaining intact internal rotators (subscapularis, latissimus dorsi, pectoralis major, teres major); however, with the loss of the posterior deltoid, the only power to external rotation comes from the infraspinatus and teres minor [1]. Furthermore, the rotational moment arms of the remaining intact rotator cuff may be reduced by the altered mechanics of RTSA [13]. Accordingly, several clinical studies have shown RTSA to be effective in improving active elevation and abduction but not external rotation $[4,22,27]$.
Despite the increasing body of literature defining the biomechanics of RTSA [1-3, 5, 12, 13, 19, 24], little is known regarding the influence individual muscle properties have on validated outcomes scores. Only a few studies [4, $11,25]$ have established an association between shoulder muscle properties and validated clinical outcomes. Greiner et al. [11] showed that the degree of postoperative fatty degeneration of the deltoid negatively correlates with functional outcomes scores of patients after RTSA. Simovitch et al. [25] and Boileau et al. [4] showed that advanced fatty infiltration of the teres minor also negatively correlates to clinical outcomes. However, to our knowledge, there have been no studies correlating preoperative quantitative muscle size and fatty infiltration with postoperative validated outcomes measures.

We therefore sought to evaluate whether there were associations between validated outcomes scores, ROM, and strength 2 years after RTSA and (1) preoperative deltoid size, (2) preoperative deltoid fatty infiltration, and (3) rotator cuff fatty infiltration observed on MR images obtained before surgery.

\section{Patients and Methods}

\section{Patients}

This study involved a retrospective analysis of patients whose clinical data were maintained in a prospective Institutional Review Board-approved outcomes registry. As part of this registry, all patients undergoing RTSA by the senior author (JMW) were asked to return postoperatively at 2 weeks, 3 months, 6 months, 1 year, and yearly thereafter for radiographic analysis and collection of outcome data. Inclusion criteria for this study were diagnosis of cuff tear arthropathy or irreparable rotator cuff tear, implantation of the same prosthetic design (Zimmer Trabecular Metal Reverse Shoulder System, Warsaw, IN, USA), minimum 2-year clinical and radiographic followup, and a preoperative MRI data set. During the reviewed period from September 2007 to March 2011, 97\% of the qualifying patients $(215 / 222)$ received this implant. Early during the study period, one patient had a different implant before the senior author (JMW) transitioned to using the current implant, and toward the end of the study period, six patients had a hybrid reconstruction using a DJO Encore ${ }^{\circledR}$ baseplate (Encore Medical LLC, Austin, TX, USA) combined with a Zimmer stem. Exclusion criteria were failed previous shoulder arthroplasty, fracture, and previous open rotator cuff surgery (to avoid confounding effects of surgical incision of the deltoid).

During the period in question, 222 patients underwent RTSA for cuff tear arthropathy or irreparable rotator cuff 
Table 1. Demographics of study patients and those lost to followup

\begin{tabular}{llll}
\hline Variable & $\begin{array}{l}\text { Patients who had RTSA during the } \\
\text { reviewed period* }(\mathrm{n}=222)\end{array}$ & $\begin{array}{l}\text { Patients with 2-year } \\
\text { followup }(\mathrm{n}=113)\end{array}$ & $\begin{array}{l}\text { Patients included in } \\
\text { the study }(\mathrm{n}=30)\end{array}$ \\
\hline $\begin{array}{l}\text { Age (years) } \\
\text { Sex }\end{array}$ & $71 \pm 10$ & $71 \pm 10$ & $71 \pm 10$ \\
$\quad$ Female & $62 \%(\mathrm{n}=137)$ & $65.5 \%(\mathrm{n}=74)$ & $73 \%(\mathrm{n}=22)$ \\
$\quad$ Male & $38 \%(\mathrm{n}=85)$ & $34.5 \%(\mathrm{n}=39)$ & $27 \%(\mathrm{n}=8)$ \\
Preoperative ASES & $30.4 \pm 17.3$ & $32.9 \pm 18.0$ & $34.7 \pm 19.8$ \\
Preoperative Constant-Murley score & $28.2 \pm 14.7$ & $29.4 \pm 15.2$ & $28.6 \pm 13.8$ \\
\hline
\end{tabular}

RTSA = reverse total shoulder arthroplasty; ASES = American Shoulder and Elbow Surgeons; *study period = September 2007-March 2011.

tear. Of those, 51\% (113/222) had 2-year followup information available. These patients were found to be similar at baseline to the overall group of patients who underwent surgery in terms of age, gender, and preoperative outcomes scores (Table 1). We surveyed that group to identify all who presented with MR images of the shoulder. During the study period, the surgeon did not obtain MRI for these indications, therefore this group represented patients who presented with MR images performed elsewhere. In total, 30 patients (eight males, 22 females; $27 \%$ of those with 2-year followup and 14\% undergoing RTSA) met these criteria and were included for analysis. These patients also were found to be similar at baseline to the overall group of patients who underwent surgery in terms of age, gender, and preoperative outcomes scores (Table 1).

MR images were collected retrospectively and therefore are not standardized. Consequently, not all patients had the correct sequences (described subsequently) for each analysis. All patients had the correct sequences for deltoid size measurements, 25 patients for fatty infiltration analysis of the deltoid, and 18 for fatty infiltration analysis of the rotator cuff muscles. The average age of the patients at the time of the procedure was $71 \pm 10$ years, and the average clinical followup was $30 \pm 7$ months. The average length of time between MRI and surgery was $4 \pm 3$ months. Our operative technique and postoperative rehabilitation protocol were described previously [16-18, 22, 28].

\section{Clinical Assessment}

Patients were examined preoperatively and postoperatively by an independent, trained clinical research nurse. Functional outcomes were measured using the Constant-Murley score [6], American Shoulder and Elbow Surgeons (ASES) total and activities of daily living (ADL) score [21], and the subjective shoulder value [8]. Patients were asked to rate their pain at its worst on a VAS from 0 (none) to 10 (maximal) [14]. ROM was measured with a goniometer and included active external rotation and active forward elevation. Active internal rotation was measured as the highest lumbar level that the patient could reach. Strength in $90^{\circ}$ abduction was measured using a hand-held digital dynamometer (Innovative Design Orthopaedics, London, UK). A chart review was performed and complication data were recorded. All outcome measures were collected and reviewed under approval of the Beaumont Health System Human Investigations Committee.

\section{Magnetic Resonance Imaging}

MRI data were collected retrospectively. Axial proton density-weighted sequences were used for cross-sectional area quantification of the deltoid muscles. All proton density data sets were acquired on a high-field MRI system. Sagittal or coronal T1-weighted, nonfat-suppressed sequences were used for fatty infiltration quantification of the deltoid, and only sagittal T1-weighted nonfat-suppressed sequences were used for fatty infiltration quantification of the rotator cuff. T1-weighted data sets also were acquired on a highfield MRI system.

\section{Muscle Size Analysis}

The cross-sectional area of the anterior deltoid, middle deltoid, and posterior deltoid were measured on the axial proton-density-weighted MRI sequences. To measure slices of consistent location, the slice with the largest humeral head and the two slices above and below were selected for analysis. The anterior, posterior, and middle deltoid were outlined using a freehand selection tool as part of a custom graphic user interface written in MATLAB $^{\circledR}(\mathrm{R} 2013 \mathrm{a}$; Mathworks, Natick, MA, USA). Outlines were drawn by one shoulder fellowship-trained orthopaedic surgeon (BPW) and two musculoskeletal fellowship-trained radiologists (SY and DRM), all blinded to patient identity and clinical outcome. Outlines were drawn in triplicate by each grader, and the triplicate measurements were averaged. The 
cross-sectional area of each muscle was calculated by converting pixel count to area in $\mathrm{cm}^{2}$. Total deltoid size was calculated as the sum of the size of each division. The use of MRI has been validated for quantitative evaluation of muscle volume [26].

Qualitative and Quantitative Muscle Fatty Infiltration Analysis

Fatty infiltration of the deltoid, supraspinatus, infraspinatus, teres minor, and subscapularis were assessed on sagittal or coronal T1-weighted nonfat-suppressed MR images. Outlines used to isolate individual muscles were made by the same, blinded investigators. Analysis of the deltoid muscle was performed with sagittal sequences using the four most lateral slices of the deltoid and with coronal sequences using the five most posterior slices of the deltoid. The rotator cuff muscles were analyzed using the slice in which the scapular spine is in contact with the scapular body and two slices lateral and medial to it. The two musculoskeletal fellowship-trained radiologists, using a 5-point scale according to the method of Fuchs et al. [7], graded fatty infiltration of each muscle qualitatively. Their scale is an MRI adaptation of the 5-point scale described by Goutallier et al. [10] (Table 2). Quantitative fatty infiltration was determined using a digital imageprocessing algorithm developed in MATLAB ${ }^{\circledR}$ (R2013a; Mathworks). This algorithm uses adaptive thresholding based on local variances in signal intensity allowing for accurate detection of muscle-fat boundaries (Fig. 1). Absolute thresholding alone was unable to account for scan-to-scan variability in pixel intensities owing to small variations in MRI sequence parameters and the decay in signal at the periphery of an MRI coil and as the distance from magnetic isocenter increases. The fraction of intramuscular fat was calculated by dividing the number of detected fat pixels by the total number of pixels in the outlined region of the muscle and results were averaged between the five analyzed slices.

\section{Statistical Analysis}

All analyses were performed using SPSS ${ }^{\circledR}$ Statistics 20 (IBM, Armonk, NY, USA). Because of the complex

Table 2. Fatty infiltration grading system of Goutallier et al.

\begin{tabular}{ll}
\hline Stage & MRI appearance \\
\hline 0 & No fatty infiltration \\
1 & Some fatty streaks \\
2 & Less fat than muscle \\
3 & Equal muscle and fat \\
4 & More fat than muscle \\
\hline
\end{tabular}

and unique nature of our data set, an a priori power analysis was not performed. However, a post hoc power analysis of our correlations showed an average of $51 \% \pm 4 \%$ (range, $51 \%-98 \%$ ) power. A ShapiroWilks test was used to test for normality. Intraobserver variations were determined with the intraclass correlation coefficient (ICC). The ICCs for intraobserver reliability were $0.946,0.943$, and 0.746 for the anterior, posterior, and middle deltoid, respectively $(p<0.001)$. Correlation of muscle cross-sectional area, subjective and quantitative fatty infiltration with postoperative outcomes measures, and improvement in outcome measures from preoperative to postoperative were analyzed with a Spearman rank correlation coefficient $(\rho)$. A Pearson's correlation test $(\mathrm{R})$ was used to analyze the correlation between subjective fatty infiltration grade and quantitative fatty infiltration. Correlation coefficients of 0.20 to $0.40,0.40$ to 0.60 , and 0.60 to 1.0 were considered mild, moderate, and strong, respectively. In all tests, p less than 0.05 was considered statistically significant.

\section{Results}

\section{Deltoid Size and Clinical Outcomes}

The cross-sectional area of each division of the deltoid and the cross-sectional area of the total deltoid was positively correlated with several functional outcomes scores and improvement in functional outcomes scores (Table 3 ). The anterior deltoid cross-sectional area $\left(8.61 \mathrm{~cm}^{2} \pm 2.97\right.$ $\mathrm{cm}^{2}$ ) had mild to moderate correlations with postoperative Constant-Murley score $\quad(67.27 \pm 13.07) \quad(\rho=0.396$; $\mathrm{p}=0.030)$, subjective shoulder value $(82.67 \pm 17.89)$ $(\rho=0.458 ; p=0.011)$, and improvement in subjective shoulder value $(50.55 \pm 32.99)(\rho=0.373 ; \mathrm{p}=0.046)$. The posterior deltoid cross-sectional area $\left(15.45 \mathrm{~cm}^{2} \pm\right.$ $7.07 \mathrm{~cm}^{2}$ ) had mild to moderate correlations with postoperative Constant-Murley score $(\rho=0.418 ; p=0.021)$, ASES $(82.64 \pm 14.25) \quad(\rho=0.369 ; p=0.045)$, ASES total $(47.92 \pm 25.25)(\rho=0.491 ; p=0.006)$ and ADL improvement $(11.50 \pm 6.62)(\rho=0.364 ; p=0.048)$, and subjective shoulder value $(\rho=0.402 ; p=0.028)$. The middle deltoid cross-sectional area $\left(5.15 \mathrm{~cm}^{2} \pm 2.74 \mathrm{~cm}^{2}\right)$ correlated only with ASES improvement $(\rho=0.401$; $\mathrm{p}=0.028)$. The total deltoid size $\left(29.21 \mathrm{~cm}^{2} \pm 11.41\right.$ $\mathrm{cm}^{2}$ ) correlated with postoperative Constant-Murley score $(\rho=0.432 ; \quad p=0.017), \quad$ postoperative ASES score $(\rho=0.377 ; p=0.040)$, ASES improvement $(\rho=0.493$; $p=0.006)$, and subjective shoulder value $(\rho=0.427$; $\mathrm{p}=0.019$ ). 

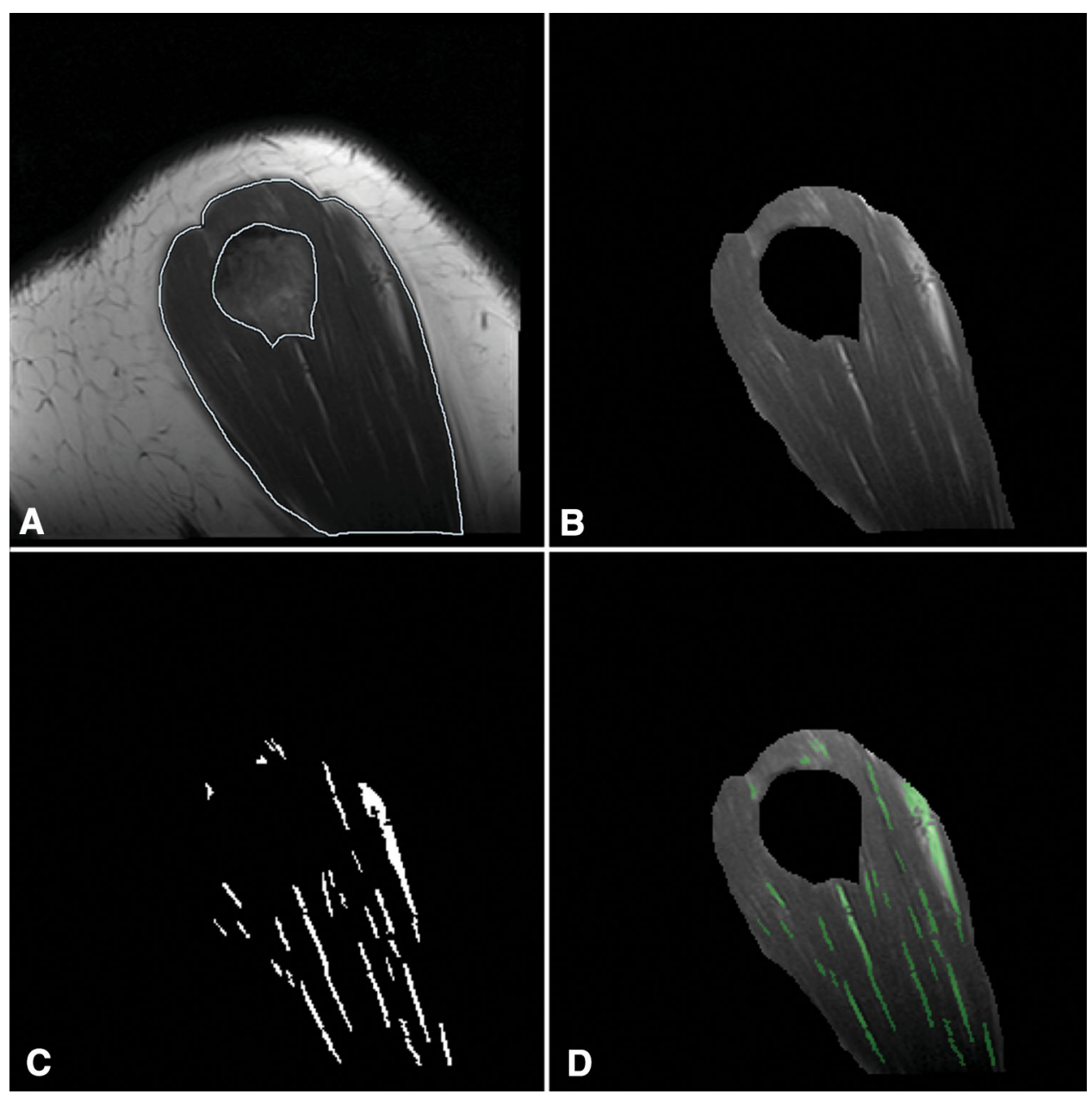

Fig. 1A-D Quantitative fatty infiltration was measured using sagittal T1-weighted MRI data. The (A) deltoid was outlined manually outlined by two musculoskeletal radiologists, and (B) the isolated muscle pixels were processed using a custom image analysis

There were no correlations between the cross-sectional area of any deltoid division with postoperative ROM or improvement in ROM (Table 4).

The cross-sectional area of the anterior and posterior deltoid and the total deltoid showed moderate correlations to postoperative strength at $90^{\circ}$ abduction ( 3.72 pounds \pm 2.99 pounds) $(\rho=0.439, p=0.019 ; \rho=0.387, p=0.042$; $\rho=0.454, p=0.015$, respectively) but not to strength improvement (Table 4).

\section{Deltoid Fatty Infiltration and Clinical Outcomes}

The Fuchs grade (Table 5) did not correlate to any postoperative functional outcomes scores, ROM, strength, or improvements in any of these variables.

However, the assessed qualitative Fuchs grade of respective muscles correlated with the quantitative fatty algorithm able to determine the differences in mean local pixel intensities to isolate (C) individual fat pixels. This processing algorithm resulted in accurate representation of the fraction of intramuscular fat, as shown by (D) overlay images.

infiltration ratio calculated with image processing (Table 6).

The quantitatively measured fatty infiltration of the deltoid had a moderate correlation with decreased postoperative ASES $(\rho=-0.401 ; p=0.047)$ (Table 7).

\section{Rotator Cuff Fatty Infiltration and Clinical Outcomes}

The supraspinatus muscle was found to have the highest degree of fatty infiltration (average, 57.4\%) as assessed by qualitative and quantitative analyses (Tables 5, 6). There were no correlations with outcomes and quantitative fatty infiltration of the supraspinatus, teres minor, or subscapularis; however, quantitative fatty infiltration of the infraspinatus correlated with decreased active external rotation $\quad\left(34.13^{\circ} \pm 16.80\right) \quad(\rho=-0.494 ; \quad p=0.037)$ (Tables 7, 8). 


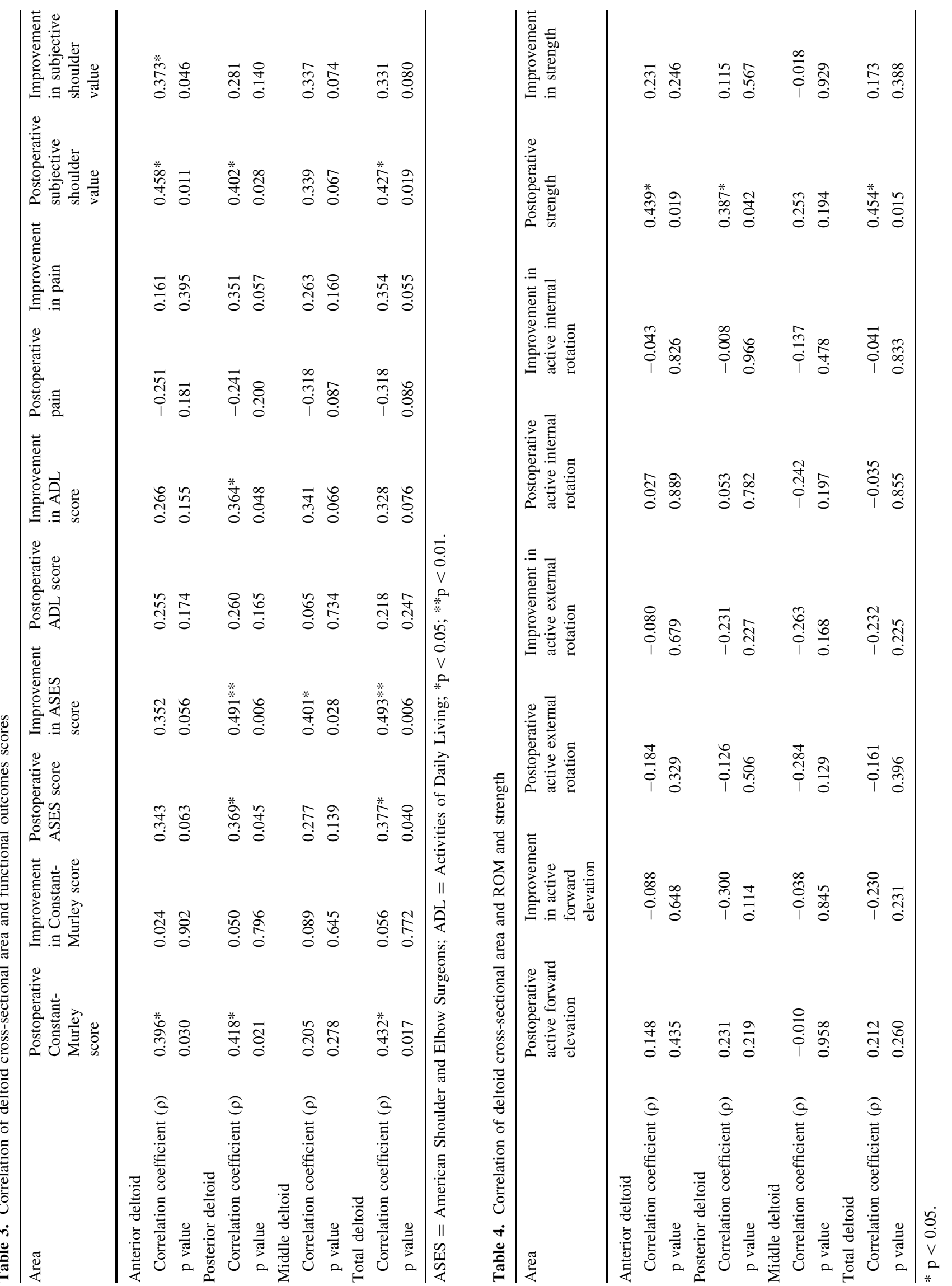


Table 5. Distribution of Fuchs grade of individual muscles

\begin{tabular}{llllll}
\hline Grade & $\begin{array}{l}\text { Deltoid } \\
(\mathrm{n}=25)\end{array}$ & $\begin{array}{l}\text { Subscapularis } \\
(\mathrm{n}=18)\end{array}$ & $\begin{array}{l}\text { Infraspinatus } \\
(\mathrm{n}=18)\end{array}$ & $\begin{array}{l}\text { Supraspinatus } \\
(\mathrm{n}=18)\end{array}$ \\
\hline 0 & 1 & 1 & 0 & 0 & $\begin{array}{l}\text { Teres minor } \\
(\mathrm{n}=18)\end{array}$ \\
1 & 17 & 13 & 4 & 2 & 4 \\
2 & 5 & 2 & 6 & 2 & 1 \\
3 & 2 & 0 & 6 & 6 & 0 \\
4 & 0 & 2 & 2 & 3 & 0 \\
Average grade & 1.32 & 1.39 & 2.33 & 3.11 & 1.06 \\
\hline
\end{tabular}

Table 6. Correlation of qualitative Fuchs score and quantitative analysis

\begin{tabular}{lclr}
\hline Muscle & Quantitative fat $(\%)$ & $\mathrm{R}$ & $\mathrm{p}$ Value \\
\hline Deltoid $(\mathrm{n}=25)$ & $7.91 \pm 4.32$ & 0.658 & $<0.001$ \\
Subscapularis $(\mathrm{n}=18)$ & $18.36 \pm 15.59$ & 0.907 & $<0.001$ \\
Teres minor $(\mathrm{n}=18)$ & $11.87 \pm 9.19$ & 0.659 & 0.003 \\
Supraspinatus $(\mathrm{n}=18)$ & $57.40 \pm 15.31$ & 0.772 & $<0.001$ \\
Infraspinatus $(\mathrm{n}=18)$ & $30.47 \pm 15.01$ & 0.780 & $<0.001$ \\
\hline
\end{tabular}

\section{Discussion}

Shoulder kinematics are changed after RTSA as the deltoid becomes the primary motor for forward elevation and abduction and the rotator cuff muscles also assume altered force vectors but remain important for internal and external rotation [1-3, 12, 13, 19, 24]. Despite a relatively thorough understanding of these biomechanical changes, it is unknown whether certain muscle properties allow for better accommodation of these changes ultimately producing improved shoulder function and an overall better clinical result. To our knowledge, there are no published studies correlating preoperative shoulder muscle size and quality with postoperative validated outcomes measures. In the current study, we found that preoperative shoulder muscle properties as seen on MR images correlate with 2-year outcomes after RTSA. Although size and fatty infiltration of the deltoid did not correlate with shoulder ROM, greater preoperative deltoid size and less fatty infiltration correlated with greater improvements in functional outcomes scores and strength. Also, fatty infiltration of the infraspinatus may negatively affect postoperative external rotation.

This study has several limitations and therefore should be considered a preliminary investigation. First, owing to the retrospective collection of MR images from multiple institutions, we were unable to standardize the imaging protocol. Despite this limitation, our image analysis algorithm had built-in functionality to minimize the effects of sequence-to-sequence variation. Second, the small proportion of available patients (14\%) who had usable MRI data is a limitation that resulted in low statistical power and the potential for selection bias. However, selection bias likely was minimized because preoperative MR images were not part of the senior author's (JMW) routine preoperative workup. In general, these patients presented with MR images that had been ordered by the patients' referring physician. This, along with comparable demographics, led us to reason that this cohort of patients was a random, albeit small, sample of the entire study population. Nonetheless, the possibility exists that these patients had advanced imaging owing to extensive disorders or questionable diagnoses. Third, this study is limited by its limited statistical analysis. Several correlations were performed, which increases the possibility of type I errors. However, when adding corrections for multiple analyses, type I errors are minimized but type II errors are exacerbated. As this is preliminary research, we chose to err on the side of a less conservative method and did not include corrections. Furthermore, several confounding patient factors exist (eg, men generally are stronger), and in the future, multivariate analyses should be performed in more highly powered studies. Finally, we recognize the potential influence of demographics and biometric variables on clinical outcomes. Although we recognize that demographic and biometric variables have confounding effects on our data set, the correlations between the muscle parameters and outcomes likely remain relevant. Significant results from our study in addition to nonsignificant trends may be further elucidated with a larger sample size and more rigorous statistical analysis.

Preoperative deltoid size was associated with several functional outcome measures, improvement in functional outcome measures, and strength. Of the three divisions of the deltoid, the posterior, followed by the anterior division, had the most positive correlations. The relative importance of the anterior and posterior deltoid over the middle deltoid may be related to muscle fiber length and orientation. The average lengths of the anterior, middle, and posterior deltoid in the native shoulder have been shown to be $144 \mathrm{~mm}$ ( $\pm 8 \mathrm{~mm}), 55 \mathrm{~mm}( \pm 9 \mathrm{~mm})$, and $178 \mathrm{~mm}( \pm 6 \mathrm{~mm})$, respectively [23]. In a RTSA, as the center of rotation is moved distal and medial, the relatively short middle deltoid 


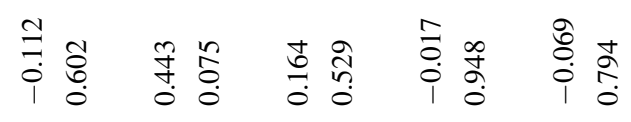

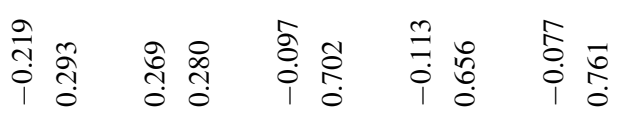

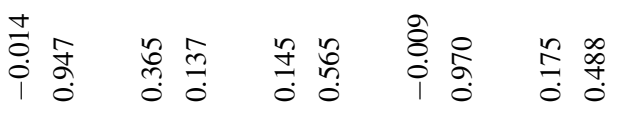

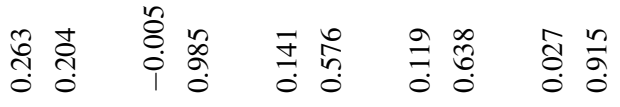

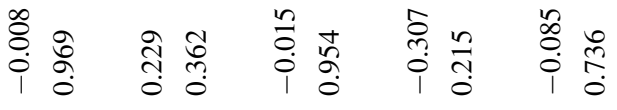

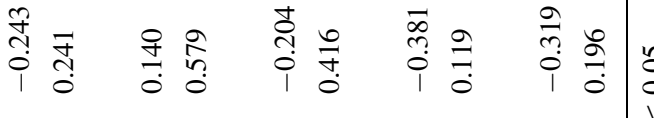

(2)

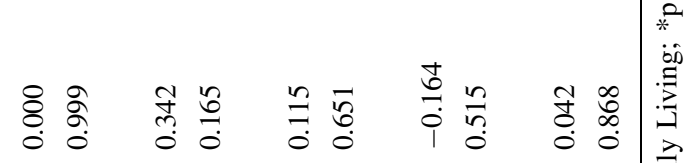

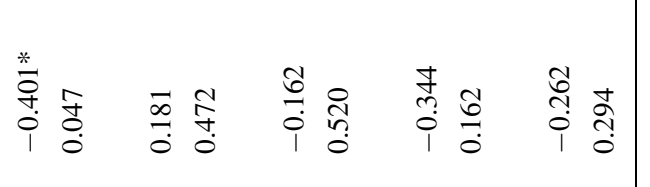

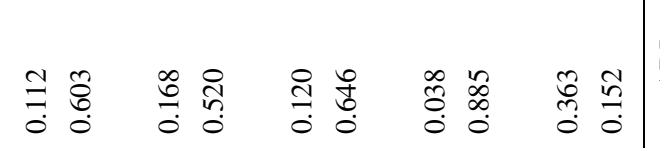

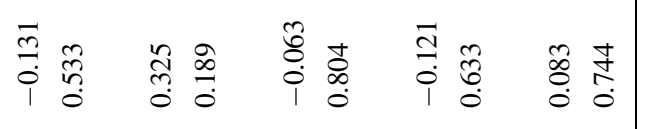

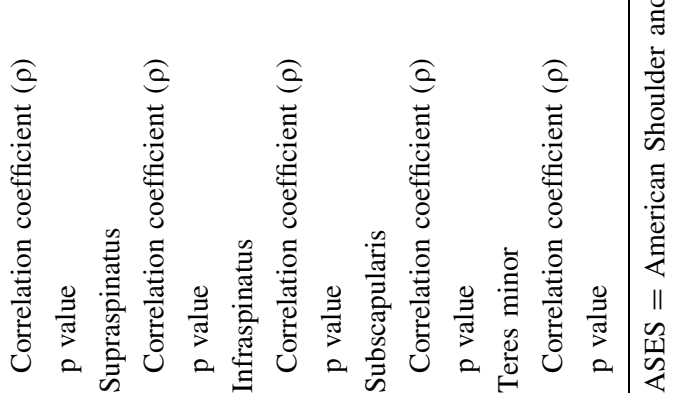




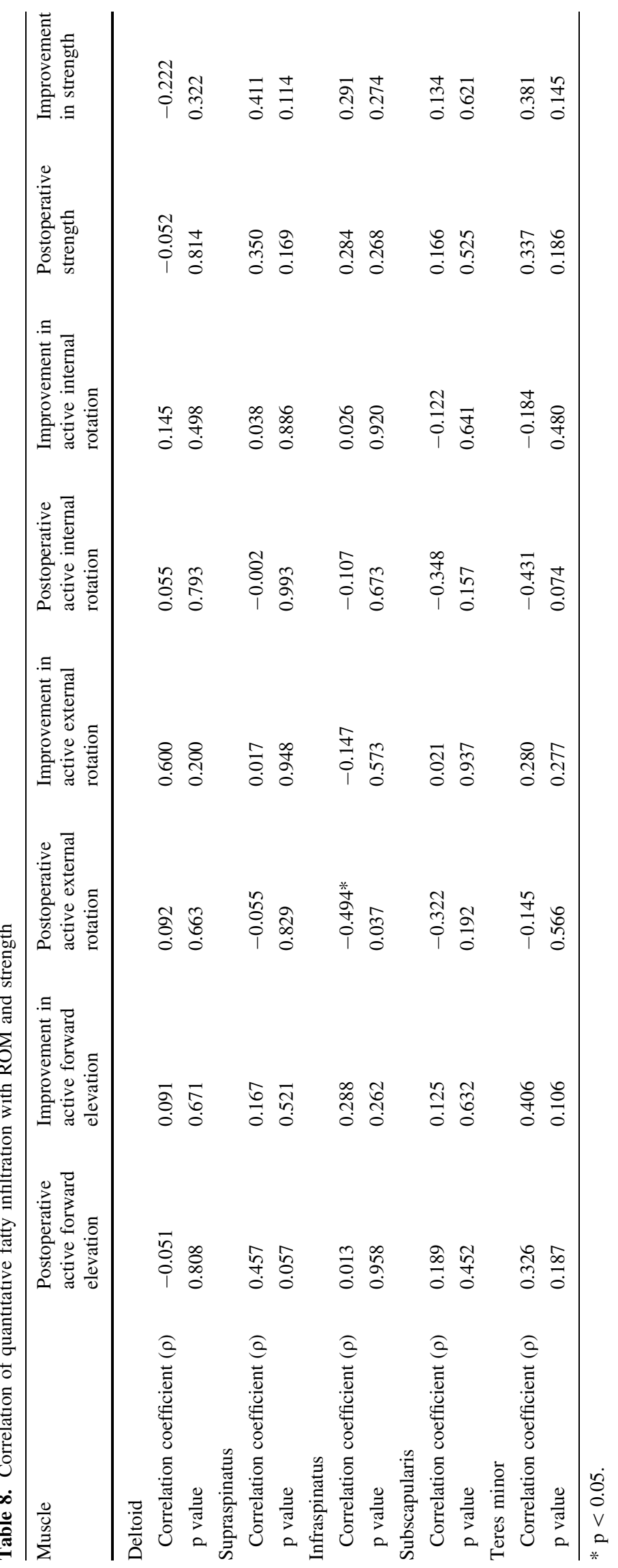


is lengthened further and may lose excursion because of being placed too high on the muscle fiber length-tension curve. Conversely, the anterior and posterior deltoid may be able to accommodate the new center of rotation not only by the longer resting length, but also by the change in muscle fiber orientation. Thus, the biomechanical changes of a RTSA may rely more on the anterior and posterior deltoid as opposed to the middle deltoid for function. Our results did not show an association between preoperative deltoid size and postoperative ROM. No correlation between deltoid size and active rotation was expected given the new role of the deltoid as primarily a forward elevator and abductor. However, lack of correlation to active forward elevation was unexpected. This may be related to the relative importance of the biomechanical advantage of the deltoid in a RTSA over the intrinsic properties of the muscle in achieving superior active forward elevation. Given that the RTSA increases the deltoid lever arm and reduces muscle effort for forward elevation and abduction $[12,19]$, deltoid size and quality may not be as important for improved active elevation as appropriate deltoid lengthening. Other studies have established the association between deltoid lengthening and active forward elevation [2, 15]. We did not assess deltoid lengthening and, therefore, cannot conclude whether it was associated with outcomes in this cohort.

In this study, deltoid fatty infiltration was associated with lower postoperative ASES scores. These results are in concordance with those of Greiner et al. [11]. They found a significant correlation between higher fatty infiltration of the deltoid and lower postoperative Constant-Murley score using postoperative MR images. Furthermore, using preoperative MR images for comparison, they found progression of fatty degeneration of the deltoid and some rotator cuff muscles after surgery [11]. The importance of muscle degeneration after RTSA remains largely unknown; however, it has been reported that muscle atrophy progression does not necessarily correlate to clinical outcome [9, 20]. Additional studies are necessary to fully characterize deltoid fatty degeneration after RTSA and the longterm effect on outcomes.

Insufficiency of the posterior rotator cuff limits active external rotation after a RTSA. In the current study, fatty infiltration of the infraspinatus correlated significantly with decreased postoperative active external rotation. Biomechanically, the inferior infraspinatus and teres minor have been shown to be the primary motors in powering active external rotation after RTSA [1]. Clinical studies have not shown fatty infiltration of the infraspinatus alone to be a risk factor for poor active external rotation after RTSA [11, 25]; however, others have shown that severe fatty infiltration of the posterior rotator cuff that extends to the teres minor results in a postoperative active external rotation deficit and lower functional outcomes [4, 25]. Boileau et al. [4], in a series of 25 patients, found severe fatty degeneration of the teres minor $(>50 \%)$ to result in poor active external rotation and lower Constant-Murley scores compared with shoulders with less than $50 \%$ fatty infiltration. Similarly, Simovitch et al. [25] found that absolute postoperative active external rotation, Constant-Murley score and improvement, and subjective shoulder value and improvement were significantly worse in patients with higher fatty infiltration grade of the teres minor. We were unable to find a negative correlation between teres minor fatty infiltration and lack of active external rotation; however, the proportion of patients showing fatty infiltration of the teres minor in our study was low, with only two patients having a Fuchs grade greater than 2. Therefore, the lack of correlation is likely a consequence of low statistical power. In addition, quantitative fatty infiltration of the subscapularis trended toward correlating with decreased active internal rotation; however, this also did not reach significance likely owing to low statistical power.

The current study is a preliminary exploration of this topic and the results suggest a potential role for preoperative advanced imaging of the shoulder in patients undergoing RTSA. Should future studies confirm the associations proposed in this study, information attained from preoperative advanced imaging would not only hold diagnostic value, but the assessment of the size and state of the deltoid and rotator cuff muscles would aid in perioperative patient counseling and surgical decision-making. Furthermore, as the knowledge and technique for assessing preoperative shoulder muscle parameters are simplified and refined, patient-specific interventions to improve or compensate for certain muscle properties may allow surgeons to maximize clinical outcomes after RTSA. Deltoid size and fatty infiltration of the deltoid and rotator cuff may hold prognostic value for patients undergoing RTSA. In the future, knowledge of these shoulder muscle properties may affect clinical and surgical decision-making.

Acknowledgments We thank the Beaumont Health System Surgical Clinical Trials Office for assistance with clinical data collection.

\section{References}

1. Ackland DC, Richardson M, Pandy MG. Axial rotation moment arms of the shoulder musculature after reverse total shoulder arthroplasty. J Bone Joint Surg Am. 2012;94:1886-1895.

2. Ackland DC, Roshan-Zamir S, Richardson M, Pandy MG. Moment arms of the shoulder musculature after reverse total shoulder arthroplasty. J Bone Joint Surg Am. 2010;92:1221-1230.

3. Ackland DC, Roshan-Zamir S, Richardson M, Pandy MG. Muscle and joint-contact loading at the glenohumeral joint after reverse total shoulder arthroplasty. J Orthop Res. 2011;29:1850 1858. 
4. Boileau P, Watkinson D, Hatzidakis AM, Hovorka I. Neer Award 2005: The Grammont reverse shoulder prosthesis: results in cuff tear arthritis, fracture sequelae, and revision arthroplasty. $J$ Shoulder Elbow Surg. 2006;15:527-540.

5. Boileau P, Watkinson DJ, Hatzidakis AM, Balg F. Grammont reverse prosthesis: design, rationale, and biomechanics. $J$ Shoulder Elbow Surg. 2005;14(suppl S):147S-161S.

6. Constant CR, Gerber C, Emery RJ, Sojbjerg JO, Gohlke F, Boileau P. A review of the Constant score: modifications and guidelines for its use. J Shoulder Elbow Surg. 2008;17:355-361.

7. Fuchs B, Weishaupt D, Zanetti M, Hodler J, Gerber C. Fatty degeneration of the muscles of the rotator cuff: assessment by computed tomography versus magnetic resonance imaging. $J$ Shoulder Elbow Surg. 1999;8:599-605.

8. Gilbart MK, Gerber C. Comparison of the subjective shoulder value and the Constant score. J Shoulder Elbow Surg. 2007;16:717-721.

9. Gladstone JN, Bishop JY, Lo IK, Flatow EL. Fatty infiltration and atrophy of the rotator cuff do not improve after rotator cuff repair and correlate with poor functional outcome. Am J Sports Med. 2007;35:719-728.

10. Goutallier D, Postel J-M, Bernageau J, Lavau L, Voisin M-C. Fatty muscle degeneration in cuff ruptures: pre-and postoperative evaluation by CT scan. Clin Orthop Relat Res. 1994;304:78-83.

11. Greiner SH, Back DA, Herrmann S, Perka C, Asbach P. Degenerative changes of the deltoid muscle have impact on clinical outcome after reversed total shoulder arthroplasty. Arch Orthop Trauma Surg. 2010;130:177-183.

12. Henninger HB, Barg A, Anderson AE, Bachus KN, Tashjian RZ, Burks RT. Effect of deltoid tension and humeral version in reverse total shoulder arthroplasty: a biomechanical study. $J$ Shoulder Elbow Surg. 2012;21:483-490.

13. Herrmann S, Konig C, Heller M, Perka C, Greiner S. Reverse shoulder arthroplasty leads to significant biomechanical changes in the remaining rotator cuff. J Orthop Surg Res. 2011;6:42.

14. Huskisson EC. Measurement of pain. Lancet. 1974;2:1127-1131.

15. Jobin CM, Brown GD, Bahu MJ, Gardner TR, Bigliani LU, Levine WN, Ahmad CS. Reverse total shoulder arthroplasty for cuff tear arthropathy: the clinical effect of deltoid lengthening and center of rotation medialization. J Shoulder Elbow Surg. 2012;21:1269-1277.

16. Kempton LB, Ankerson E, Wiater JM. A complication-based learning curve from 200 reverse shoulder arthroplasties. Clin Orthop Relat Res. 2011;469:2496-2504.
17. Kempton LB, Balasubramaniam M, Ankerson E, Wiater JM. A radiographic analysis of the effects of glenosphere position on scapular notching following reverse total shoulder arthroplasty. $J$ Shoulder Elbow Surg. 2011;20:968-974.

18. Kempton LB, Balasubramaniam M, Ankerson E, Wiater JM. A radiographic analysis of the effects of prosthesis design on scapular notching following reverse total shoulder arthroplasty. $J$ Shoulder Elbow Surg. 2011;20:571-576.

19. Kontaxis A, Johnson GR. The biomechanics of reverse anatomy shoulder replacement-a modelling study. Clin Biomech (Bristol, Avon). 2009;24:254-260.

20. Mellado JM, Calmet J, Olona M, Esteve C, Camins A, Pérez Del Palomar L, Giné J, Saurí A. Surgically repaired massive rotator cuff tears: MRI of tendon integrity, muscle fatty degeneration, and muscle atrophy correlated with intraoperative and clinical findings. AJR Am J Roentgenol. 2005;184:1456-1463.

21. Michener LA, McClure PW, Sennett BJ. American Shoulder and Elbow Surgeons Standardized Shoulder Assessment Form, patient self-report section: reliability, validity, and responsiveness. J Shoulder Elbow Surg. 2002;11:587-594.

22. Nolan BM, Ankerson E, Wiater JM. Reverse total shoulder arthroplasty improves function in cuff tear arthropathy. Clin Orthop Relat Res. 2011;469:2476-2482.

23. Peterson SL, Rayan GM. Shoulder and upper arm muscle architecture. J Hand Surg Am. 2011;36:881-889.

24. Schwartz DG, Kang SH, Lynch TS, Edwards S, Nuber G, Zhang LQ, Saltzman M. The anterior deltoid's importance in reverse shoulder arthroplasty: a cadaveric biomechanical study. $J$ Shoulder Elbow Surg. 2013;22:357-364.

25. Simovitch RW, Helmy N, Zumstein MA, Gerber C. Impact of fatty infiltration of the teres minor muscle on the outcome of reverse total shoulder arthroplasty. J Bone Joint Surg Am. 2007;89:934-939.

26. Tingart MJ, Apreleva M, Lehtinen JT, Capell B, Palmer WE, Warner JJ. Magnetic resonance imaging in quantitative analysis of rotator cuff muscle volume. Clin Orthop Relat Res. 2003;415:104-110.

27. Werner CM, Steinmann PA, Gilbart M, Gerber C. Treatment of painful pseudoparesis due to irreparable rotator cuff dysfunction with the Delta III reverse-ball-and-socket total shoulder prosthesis. J Bone Joint Surg Am. 2005;87:1476-1486.

28. Wiater BP, Boone CR, Koueiter DM, Wiater JM. Early outcomes of staged bilateral reverse total shoulder arthroplasty: a casecontrol study. Bone Joint J. 2013;95:1232-1238. 